\title{
THE PROCESS AND PURPOSE OF READING
}

Rohib Adrianto Sangia

Abstract: This paper as a compulsory assignment in Applied Linguistics subjects describes and explains the theories in helping people which deal with language to make the language activities more effective. This opinion and purposes in this material hopefully can help those people which is working or doing the business in language teaching especially teaching for reading skills. Since this paper is emphasizing the language teaching particularly in teaching reading skills, the theories that will be presented comes from H.G Widdowson from his journal "The Process and Purposes of Reading” in Exploration in Applied Linguistics.

Keywords: Reading Process, Reading Purpose, Teaching Reading, Encoding, Decoding

This paper is containing several parts around the reading activity theories. The beginning starts with the definition about reading itself as the center of the topic and as one of fundamental skills which most used by people. Next part describes the process of reading which is a part of teaching reading in order to make a meaningful learning for the students to get better result in students' understanding of the text. Discussing text as medium of interaction between writer and reader will be discussed in the next section. It is followed by clear explanation of encoding and decoding process and adds with teaching implementation. Finally it presents the purpose of reading and suggestions in doing reading activities for the language teachers and students.

\section{Definition of Reading}

Reading is one of skill in the language that needs to be considered. Skilled reading makes students better understand all the material taught. This indicates that the subjects read in the field of Indonesian studies should receive greater attention.

Reading as one aspect of the four language skills, plays an important role in language teaching. Said to be important because, in addition to teaching listening, speaking, and writing. Reading skills is one very powerful tool for obtaining a wide range of specific information, including science and technology. Therefore, reading is a basic requirement for an advanced society. Similarly, in education, read a very influential role in student performance. It can be proved that the higher the reading understanding of the students, gains the higher the knowledge they had. Thus the interest in reading and reading skills 
students need to be grown as early as possible, so that students can understand the role and function of reading. Good communication tool and as a learning tool to develop knowledge and skills expand horizons. It can be said that the students who have literacy levels higher will be easier to acquire science and technology contained in the print media or the media write. Based on this, the government has made efforts to cultivate the habit of reading among students and the outside community, for example by setting up school libraries and public libraries, holding book fairs, and seminars that aim to stimulate students' interest and society in general.

Reading is a selective process. It involves partial use of available minimal language cues selected from perceptual input on the basis of the reader's expectation. As this partial information is processed, tentative decisions are made to be confirmed, rejected, or refined as reading progresses (Goodman, 1967: 128).

Widdowson (1979: 169) claims that the definition of reading that Goodman (1967: 128) purposed describes the general process which reveals that reading is basically a specific comprehension of discourse strategy. This definition gives the impression that reading is reaction to meanings which translated explicitly from the text. Since it is explicitly converted from text, it results the consequence that there are many assumption is using, so that the entire understanding from the content of text cannot always be fully seized.

Reading skills is a complex activity that involves a variety of factors that come from within the reader and external factors. In addition, reading skills can also be regarded as a product of human kind's ability to learn from the environment, and not a capability that is instinctive or inborn instinct. Therefore, the process of reading is carried out by an adult (to read) is a business process and produce something through the use of specific capital.

\section{The Process of Reading}

Reading is a complex cognitive process of decryption signs in order to create or originate meaning (Schulz, Baker, Purcell, Association, and Conference, 1977: 30). It is a way of language acquisition, communication, and sharing information and ideas. Like all languages, it is a complex interaction between text and reader which is formed by a previous reader knowledge, experience, attitude, and language community which is culturally and socially situated. Reading process requires continuous training, development, and improvement. Besides, reading requires creativity and critical analysis. Commercials literature make business with each piece, congenital deviate from the literal words to create an image that makes sense to them in an unknown place it describes. Because reading is a 
complex process, cannot be controlled or restricted to one or two interpretations. There is no concrete law in reading, but allows the reader to escape to produce their own products introspectively. This encourages deep exploration of text for interpretation (Certeau, 2002: 170).

In reading activity is an aspect of language behavior with the apprehension of general process of interpretative which is covered communicative activity. It means that in reading, meaning is created by the reader base on of textual hints. This interpreting activity is not exclusive from the reading, but a packet with reading because reading is practical reasoning process that results the derived of meaning. In the process of deriving the meaning, the readers cannot create and complete to get the full meaning, but the meaning that reader gets only the meaning which is sufficient for reader needs. As conclusion, the process of reading involved how meaning is negotiated in discourse (Widdowson, 1979: 168).

Hereafter, Reading activity is a process that drives two disparate levels of mental activity. The first activity is dealing with immediate apprehension of information and the other is related with the discrimination of this information into patterns of conceptual significance (Widdowson, 1979: 168). In process of reading, the reader not only creates meaning but also meanings should be negotiated in discourse as process of reading strategy. Reading should not be reaction to meaning but as interaction between writer and reader mediated through the text. Thus, how to make an efficiency reading depends on how effective the text is (Widdowson, 1979: 169).

\section{Text as Medium of Interaction}

As mentioned by Widdowson (1979: 169) that reading is considered not as response to the text but as interaction between writer and reader mediated with text. Since it is an interaction, there should be adjusted some notion of interaction. The analogy of interaction simply can be looked in a spoken conversation. This conversation presents an obvious of negotiation of meaning through two-way communication. It is clear that the interlocutors send the complete meaning together with watchful accuracy or give utterance to critical analysis.

In spoken discourse, the interlocutors simply concerned maintaining interaction without aware with what actually being said since the meaning which is delivered quite commonly vague, imprecise, and insignificant. The interaction in here is using co-operative 
principle (Grice, 1975: 45). To get pleased interaction between interlocutors, sometimes there is some exchange for little information which needs. The interlocutors are basically worried in maintaining the interaction without concerning what is essentially being talked. The interaction is being significant when it gives the speaker and hearer with set of direction to where they can find and create meaning for themselves (Widdowson, 1979: 170).

The application of co-operative principle used in written discourse where the reader and the writer are involved in a communication where the language as a sign is practiced. Written discourse as non-reciprocal has certain important consequence for how to realize the interaction. Written discourse is created and expected in objectivity from a direct context of utterance and this can easily misinformed into concerning it as a drastically dissimilar manner of communication from spoken discourse (Widdowson, 1979: 172).

\section{Encoding and Decoding Process}

Decoding means translating written words into the sounds and meanings of spoken words (often silently). Encoding, or spelling, is the reverse process. The skills used in encoding are usually developed alongside decoding skills and reflect similar learning. Decoding is an essential skill for reading. Decoding is not enough in itself to enable comprehension, but to be a good reader it is necessary to be a good decoder. To easily read the texts in their everyday lives, adults need to be able to decode unfamiliar words without having to think about it. Encoding is about providing the direction to addressee and decoding is dealing with following the direction from addresser (Widdowson, 1979: 170).

The encoding process is correspondingly vague and estimated and it is impossible to recuperate comprehensive meaning from a text. The encoding process is not as the construction of communications, in principle complete and self-contained, but as the creating of a set of directions. These directions indicate to the decoder where the reader must look in the abstract world of his experience and knowledge for the encoder's sense. The encoder depends on the active contribution of the decoder and the decoder is successful in his comprehension to the range to be understood the directions and is accomplished of carrying them out (Widdowson, 1979: 169).

\section{Teaching Implementation}

In the teaching of reading and writing, there is an obligation to make the learner conscious first of the principally inexact character of communication over natural language. Most of teacher boosts students to have confidence in that exact meanings can be fully 
recoverable from texts, that texts will produce their total content if they are analyzed in adequate detail. Sometimes teachers avoid discouraging a normal use of natural language and refuting students' access to their own abstract world which alone confirms that reading will, in any actually significant sense, be meaningful. Teachers should make it obvious that the individuality of written discourse from a direct context does not make it any less interactive as a mode of communicating (Widdowson, 1979: 172).

By using Bronowski (1960) text, Widdowson (1979) proposed to the teacher conducts to decompose a writing text into parts of utterance and makes students ask the question which is the answers which are reconstruct in sequences for letter to be converted into paragraphs to the original passage in the written language. The next figures will show how easily one initial statements can develop different paragraph. They can be happened depend on what kinds of directions are prerequisite to different interlocutors.

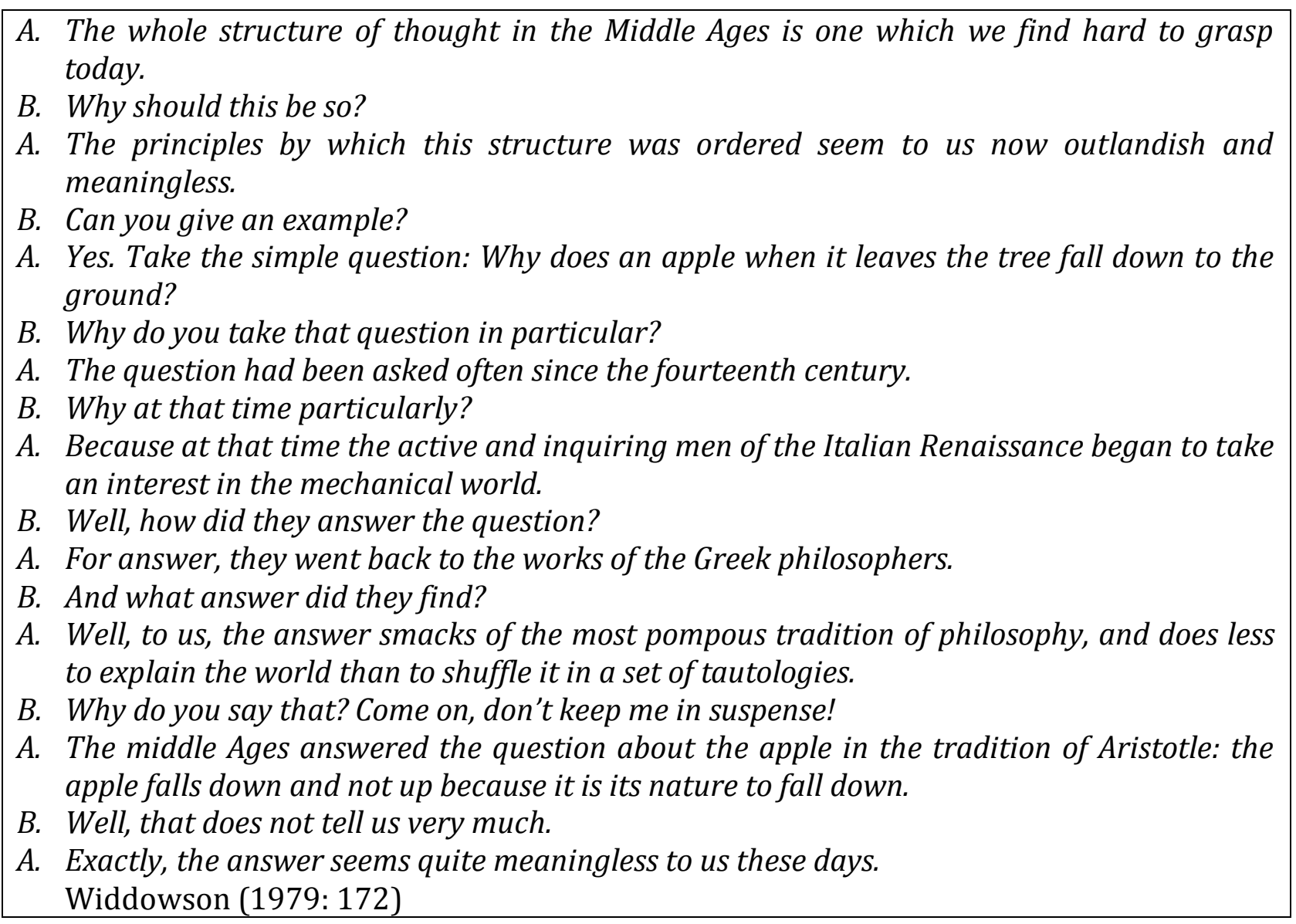

The communication above can be converted to the passage in Bronowski, which writes as follows: 
The whole structure of thought in the Middle Ages is one which we find hard to grasp today. It was orderly structure, but the principles by which it was ordered seem to us now outlandish and meaningless. Take such a simple question as that which is said to have turned Newton's mind to the problem of gravitation: Why does an apple when it leaves the tree fall to the ground? The question had been asked often since the fourteenth century, when the active and inquiring men of the Italian Renaissance began to take an interest in the mechanical world. For answer, they went back to one of the great re-discoveries of the Arabs and the Renaissance, the works of the Greek philosophers. To us, this answer smacks of the most pompous tradition of philosophy, and does less to explain the world than to shuffle it in a set of tautologies. For the Middle Ages answered the question in the tradition of Aristotle: the apple falls down and not up because it is its nature to fall down.

Bronowski (1960: 26)

On the other hand, the passage above is not only an abstract communication that could be developed from Bronowski's opening statements. Another alternative is proposed by Widdowson (1979: 173) as followed:

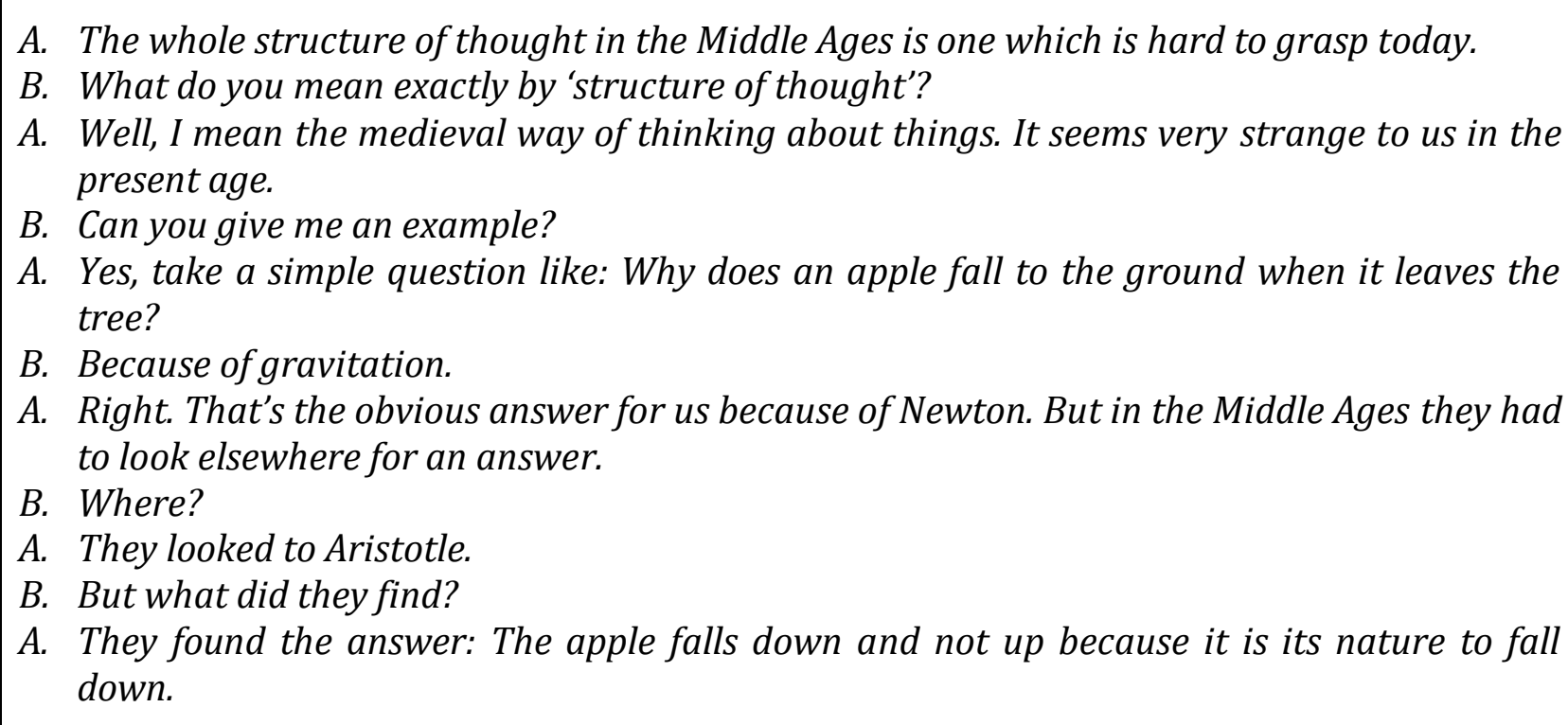

Conversation above might be transformed into a written discourse to produce the following article:

The whole structure of thought in the Middle Ages is one which we find hard to grasp today. The medieval way of thinking about things seems very strange to us in the present age. Consider, for example, the simple question: Why does an apple fall to the ground when it leaves the tree? Because of Newton, the answer to us is obvious: gravitation. But the Middle Ages had to look elsewhere for an answer. They turned to Aristotle, and the answer they found was: The apple falls down and not up because it is its nature to fall down Widdowson (1979: 174)

Another strategy in presenting reading as an interactive process might be succeeded by considering previous example. 


\section{Purpose of Reading and Suggestion}

In reading activity, teacher and learners catch the material in the process of direct communication. It means that one function of reading has been fulfilled. The reader categories out what is simply enabling from what is wished to take as a permanent addition to his domain of understanding and capability. The actual purpose in reading is not humbly to occupy in a communication but to develop from this communication to something which endures or spreads the reader conceptual world.

If the interaction between reader and writer is effective, so that there is close relationship between them, then there will be settlement on relative implication. But the reader is always possible to enforce his own allowance on the information he obtains. The reader may then be indicted of missing the point or taking remarks out of context. As if abstract worlds do not match, there can never be a precise equivalence of encoder's and decoder's meanings so an interaction can be estimated naturally.

In classroom activity, learners rarely have to use the information what they get, whichever within an interaction process to accommodate entrance to the most salient directions towards meaning or to follow these directions into their own abstract worlds. Reading is characterized as a culmination in itself, an activity that has no implication to actual knowledge and experience will have no real significance.

Furthermore, this kind of problem can be solve with suggestion by Widdowson (1979: 175). By making the learners conscious, over involvement in the kind of interactive drills earlier, with much written discourse serves a basically facilitating function. Trying to encourage learners to relate what they read to their own world of knowledge and experience by selecting reading material that is likely to request to their interests, but there is no point in doing this unless teachers also ensure that their interests are really betrothed by allowing them the same freedom of interpretation that is practiced by the readers. The texts should be transformed into discourse and the language put to creative use.

\section{Conclusion}

Reading is a selective progression by tentative decisions are made to be confirmed, rejected, or refined, which implicates part use of available minimal language prompts designated from perceptual input base on the reader's hope. Reading is a reasoning activity whereby the reader creates meaning on the basis of textual clues. 
In process of reading, the reader not only creates meaning but also meanings should be negotiated in discourse as process of reading strategy. Reading should not be reaction to meaning but as interaction between writer and reader mediated through the text. Process of reading involves two different levels of mental activity. The first is dealing with the immediate apprehension of information and the second with the discrimination of this information into patterns of conceptual significance.

Written text is non-reciprocal, so the interaction between reader and writer which use language as a clue to correspondence conceptual or abstract worlds. The writer needs to assume the roles both of addresser and addressee so he can incorporate the interaction as encoding process itself. The writer makes the judgments about the reader's possible reaction and anticipate any difficulties by providing understanding directions to the reader.

The actual purpose in reading is not humbly to occupy in a communication but to develop from this communication to something which endures or spreads the reader conceptual world.

\section{REFERENCES}

Bronowski, Jacob. 1960. The common sense of science. Harmondsworth: Penguin Books.

Certeau, Michel de. 2002. The practice of everyday life. Berkley: University of California Press.

Goodman, Kenneth S. 1967. Reading: A psycholinguistic guessing game. Literacy Research and Instruction Vol. 6 No. 4, 126-135.

Grice, HP. 1975. Logic and Conversation. In J. Morgan \& P. Cole (Eds.), Syntax and Semantics (Vol. 3 : Speech acts, pp. 41-57): Academic Press, New York.

Schulz, R.A., Baker, R.E., Purcell, J.M., Association, Ohio Modern Language Teachers, and Conference, Central States. 1977. Personalizing foreign language instruction: learning styles and teaching options : selected papers from the 1977 joint meeting of the Central States Conference and the Ohio Modern Language Teachers Association: National Textbook Co.

Widdowson, H. G. 1979. The process and purpose of reading Explorations in applied linguistics (pp. 168-176). Oxford: Oxford University Press. 